\title{
Reliability of Lagged Poincaré Plot parameters in ultra-short Heart Rate Variability series: Application on Affective Sounds
}

\author{
M. Nardelli ${ }^{1}$, A.Greco ${ }^{1}$, J.Bolea ${ }^{2,3}$, G. Valenza ${ }^{1}$, E.P. Scilingo ${ }^{1}$, R.Bailón ${ }^{2,3}$
}

\begin{abstract}
The number of studies about ultra-short cardiovascular time series is increasing because of the demand for mobile applications in telemedicine and e-health monitoring. However, the current literature still needs a proper validation of heartbeat nonlinear dynamics assessment from ultra-short time series. This paper reports on the reliability of the Lagged Poincaré Plot (LPP) parameters - calculated from ultra-short cardiovascular time series. Reliability is studied on simulated as well as on real RR series. Simulated RR series are generated and LPP parameters estimated for ultra-short time series (from 15 to 60 s) are compared to those estimated from 1 hour. All LPP parameters estimated from time series longer than $35 \mathrm{~s}$ presented a Spearman's correlation coefficient higher than 0.99. RR series acquired from 32 healthy subjects during 5-minutes resting state sessions are used to test the LPP approach in experimental data. The usefulness of ultra short term parameters in real data is accomplished also studying their ability to discriminate positive and negative valence of auditory stimuli taken from the International Affective Digitized Sound System (IADS) dataset. The achieved accuracies in the recognition of elicitation along the valence dimension, using only the LPP parameters, were of $77.78 \%$ for 1 minute 28 second series, and of $79.17 \%$ for 35 second series.
\end{abstract}

\section{INTRODUCTION}

It is known that Heart Rate Variability (HRV), extracted from the electrocardiographic (ECG) signals, is a promising clinical tool to investigate, in a noninvasive way, sympathetic and parasympathetic dynamics of the autonomic nervous system (ANS) [1], [2].

Although long-term HRV analysis (24 hours) has been proven to be useful for prediction of several physical and psychological diseases, e.g. myocardial infarction [3] and depression [4], it is also associated to low reproducibility and high nonstationarities [5]-[7]. Short-time analysis of HRV (5 minutes) has been proposed to overcome the non-stationarities, to be less influenced by recording conditions and suitable for the study of short time ANS response to stimuli in and outside clinical settings [1], [8], [8]-[10].

Due to the diffusion of telemedicine and e-health, the demands of ultra-short term monitoring (less than 5 minutes) of HRV is significantly increasing, especially for its usefulness in mobile

This work was supported by project TIN2014-53567-R (Spain), by Government of Aragón and European Social Fund (EU), by CIBER (Spain) and by European Union Seventh Framework Programme FP7/2007-2013 of the project "WEARHAP".

${ }^{1}$ Department of Information Engineering \& Research Centre E. Piaggio, University of Pisa, Via G. Caruso 16, Pisa, Italy

${ }^{2}$ BSICoS Group, Aragón Institute of Engineering Research (I3A), IIS Aragón, University of Zaragoza, Zaragoza, Spain

${ }^{3}$ CIBER of Bioengineering, Biomaterials and Nanomedicine (CIBER BBN), Madrid, Spain

*Corresponding author e-mail: mimma.nardelli@for.unipi.it applications [11], [12]. In e-health monitoring, in fact, the conventional 5 minutes recordings might be unsuitable, due to real time requirements. A relevant and increasingly studied use of ultra-short HRV series is the analysis of psychological state conditions, such as mental stress [11], [13], [14] or emotional arousal [15], [16].

Several previous studies investigated the reliability of the HRV parameters extracted from ultra-short term series in order to test their prognostic significance in patients with cardiac risk factors [17], or to check the post-exercise heart rate recovery in athletes [18], [19].

Studies reporting the reliability of HRV parameters calculated from ultra-short time series were promising, especially for time-domain parameters, such as RMSSD [20], [21] and SDNN [17]. However the extrapolation of these results face some limitations. For instance, the differences in studied populations, including healthy subjects as well as patients, and the aim of the study, which includes identification of cardiac diseases as well as assessment of physiological response to physical/mental stress, or to several arousal stimulations.

There are few studies in the literature that explore the behaviour of features belonging to nonlinear analysis methods in ultra-short time windows. For example, it has been suggested that record lengths of $10^{m}-20^{m}$ (where $m$ is the embedding dimension) are needed to estimate Approximate Entropy (ApEn) and Sample Entropy (SampEn) [22]. McNames et al. studied the reliability of $11 \mathrm{HRV}$ parameters as a function of the duration of the windows extracted from 54 long-term ambulatory ECG recordings [23]. They found that ApEn, the only nonlinear parameter analyzed, was the most unreliable metric of the HRV measures tested in this study.

Here we present a study about the reliability of the parameters extracted from the Lagged Poincaré Plot (LPP) [24], [25], a geometric method taken from chaos theory, which allows a beatto-beat analysis of HRV, in order to observe patterns belonging to nonlinear processes, not detectable with techniques in the time and frequency domains. Some previous works showed that this approach allows to distinguish, better than linear methods, the changes in ANS dynamics in conditions such as congestive heart failure [26] and in patients with chronic renal failure [27].

One novelty of this work is that reliability of ultra short term $\mathrm{HRV}$ parameters is done on simulated as well as on real RR series. Simulated RR series allow to test the reliability of the method under the same conditions for all time intervals, which can not be guaranteed in real data. The reliability of ultra short term parameters in real data is accomplished through statistical analysis methods using sessions of five-minute resting state, but also studying their ability to discriminate positive and neg- 
ative valence of auditory stimuli taken from the International Affective Digitized Sound System (IADS) dataset [28]-[30].

\section{Materials And Methods}

\section{A. Lagged Poincaré Plot}

The Poincaré Plot (PP) technique consists of a scatterplot generated by each RR interval against the immediately previous RR interval $\left(R R_{n+1}\right.$ and $\left.R R_{n}\right)$. In the LPP method, the points with coordinates $R R_{n}$ and $R R_{n+M}$ are represented in the scatterplot, where $M$ is the lag. [24], [25], [31]. From each series we extracted the normally used PP indexes:

- SD1: the standard deviation of the points related to the direction perpendicular to the line-of-identity $R R_{n+M}=$ $R R_{n}$ [32]. It describes the short-term dynamics of HRV in the analyzed time interval.

- SD2: the standard deviation that describes the long-term dynamics and measures the dispersion of the points along the identity line.

- $\operatorname{SD} 12$ ( $S D 12=S D 1 / S D 2)$ : the ratio between SD1 and $\mathrm{SD} 2[33]$.

- $\mathrm{S}(S=\pi \times S D 1 \times S D 2)$ : the area of an imaginary ellipse with axes SD1 and SD2 [33], [34].

- $\operatorname{SDRR}\left(S D R R=\frac{1}{\sqrt{2}} \sqrt{S D 1^{2}+S D 2^{2}}\right)$ : an approximate relation indicating the variance of the whole HRV series [34].

For each parameters we considered values calculated from the LPP with a lag range from 1 to 10 [35].

\section{B. Data used in the study}

1) Synthetic data: Synthetic RR series were generated through the IPFM model [36]. This model reproduces the heartbeat occurrence times from a modulating signal $m(t)$, which represents the behavior of the ANS, and it is assumed to be band-limited and less than one. The beat trigger impulse is generated when the integral of this function reaches a threshold, according to the following equation:

$$
k=\int_{0}^{t_{k}} \frac{1+m(t)}{T} d t
$$

where $k$ is an integer that represents the number of the $k-t h$ beat and $t_{k}$ is the occurrence time of the $k-t h$ beat, $T$ is the mean of the RR intervals series. Modulating signal $\mathrm{m}(\mathrm{t})$ is generated using an autoregressive moving average model, whose input parameters are the frequency and the power of the dominant peak in the LF (low frequency, 0.04-0.15 Hz) and $\mathrm{HF}$ (high frequency, 0.15-0.4 Hz) bands, respectively [37]. In this study the frequency of the peaks were chosen in random way in the range of the two main bands (LF and HF) and their power values were within the normal range reported in literature for healthy people (a median value of $81 \mathrm{~ms}^{2}$ and an interquartile range of $116 \mathrm{~ms}^{2}$ ) [38], [39]. A total number of 1.200 realizations of the modulating signal $\mathrm{m}(\mathrm{t})$ were generated. The parameter $\mathrm{T}$ was set to 1 second for all the realizations. In this way we generated 1200 simulated RR series of 3595 samples each (in order to have the same values of samples referred to signals of 1 hour).
These simulated RR series are analyzed in 50\% overlapped windows considering different window lengths, ranging from 15 samples to the total length in steps of 5 samples. For each window length, the LPP parameters value was estimated as the mean in all the windows of the same length.

2) Experimental data: To verify the validity of LPP parameters extracted from ultra-short windows in experimental data, we analyzed the RR series recorded into two conditions: during a 5-minutes resting state protocol and during an experiment of emotional acoustic stimulation. During both the experimental protocols, the ECG was continuously acquired, following the Einthoven triangle configuration, by means of a dedicate hardware module, i.e., the ECG100C Electrocardiogram Amplifier from BIOPAC inc. with a sampling rate of $500 \mathrm{~Hz}$. To obtain the RR series from the ECG, we used the automatic algorithm developed by Pan-Tompkins [40]. Artifacts and ectopic beats were corrected through the use of Kubios HRV software [41].

Thirty-two healthy subjects, aged from 21 to 35, participated as volunteers in the resting-state experiment. According to the self-report questionnaires, none of them suffered from any cardiovascular, mental or chronic disease. Participants were informed about the protocol and about the purpose of the study. During the experiment, subjects were seating in a comfortable chair, in a controlled environment. The participants were asked to be in resting state for five minutes while their eyes were closed. The RR series have been analyzed in $50 \%$ overlapped windows considering different window lengths, ranging from 15 to 180 samples (the first three minutes) in steps of 5 samples. For each window length, the LPP parameters value was estimated as the mean in all the windows of the same length.

Concerning the emotional acoustic stimulation we used the same stimulation protocol which we proposed in a previous work [42], which was approved by the local ethical committee. Twenty-seven healthy subjects, aged from 25 to 35, participated as volunteers. Through self-report questionnaires, we realized that none of them was affected by partial or full incapability of hearing and none of them suffered from any cardiovascular, mental or chronic disease.

During all the duration of the protocol, participants were seated alone in a controlled environment while listening to the IADS sounds, by using headphones, with closed eyes. The IADS sounds are standardized in the values of arousal, i.e. the intensity of the evoked emotion, and valence, i.e. the pleasantness-unpleasantness of the sensation, following the bidimensional model of emotions proposed by Russell [43], [44] and called Circumplex Model of Affect (CMA). Within each arousing session in the protocol, the acoustic stimuli were selected to have Low-Medium (L-M) ratings for negative valence and Medium-High (M-H) ratings for positive valence. These levels were set according to the IADS valence and arousal scores reported in Table I. The affective elicitation was comprised of 10 sessions: an initial resting session of 5 minutes and four arousal sessions alternated with neutral sessions. Within each arousing session, the acoustic stimuli were selected to have negative valence for the first part of the session and positive valence for the last half. The neutral 
Table I

RATING OF IADS SOUNDS USED IN THIS WORK

\begin{tabular}{|c|c|c|c|c|}
\hline Session & Valence Rating & Valence Range & Arousal Rating & Arousal Range \\
\hline \hline Neutral & $5.915 \pm 0.68$ & $4.34 \div 6.44$ & $3.47 \pm 0.175$ & $2.88 \div 3.93$ \\
\hline Arousal 1 & $/$ & $3.54 \div 7.51$ & $4.60 \pm 0.21$ & $4.03 \div 4.97$ \\
\hline Arousal 2 & $/$ & $2.46 \div 7.78$ & $5.42 \pm 0.22$ & $5.00 \div 5.89$ \\
\hline Arousal 3 & $/$ & $2.04 \div 7.90$ & $6.48 \pm 0.25$ & $6.00 \div 6.99$ \\
\hline Arousal 4 & $/$ & $1.57 \div 7.67$ & $7.32 \pm 0.22$ & $7.03 \div 8.16$ \\
\hline
\end{tabular}

session had a duration of 1 minute and 28 seconds, while the four arousal sessions had durations of more than 3 minutes with a difference which was due to the different length of acoustic stimuli having the same range of positive and negative valence.

In this work we considered two ultra-short time windows to extract HRV parameters, in the first time with the duration of 1 minute and 28 seconds and in the second time with a length of 35 seconds. The first time duration, i.e. 1 minute and 28 seconds was chosen due to the length of the neutral session of the protocol [42]. The windows of 1 minute and 28 seconds were selected at the beginning and at the end of each arousal session. Then we selected the second length, i.e. 35 seconds, which is a duration of resting state tested in our previous work, where we studied the autonomic response to haptic stimulation [16]. Four windows of 35 seconds were obtained by each window of 1 minute and 28 seconds considering an overlap of 17.5 seconds. Then, the mean of the parameter values in the four 35 second windows was computed so each arousal session was characterized by two values, one for the negative and the other for the positive valence.

From the RR series of both window lenghts the five parameters of LPP described in Section II-B1 were computed for ten values of lag, as well as the main HRV parameters in the timedomain: the mean of RR intervals (mean RR), the standard deviation (std RR), the square root of the mean of the sum of the squares of differences between subsequent RR intervals (RMSSD), the integral of the probability density distribution (that is, the number of all RR intervals) divided by the maximum of the probability density distribution (HRV tiangular index), the triangular interpolation of RR interval histogram (TIRR). Frequency parameters of HRV were obtained from the power spectral density (PSD) of the modulating signal $\hat{m}(t)$, obtained from the beat occurrence times, $t_{k}$, using the IPFM model [45]. PSD was estimated using Welch's periodogram and the power in the LF and HF bands (see Section II-B1) were computed, together with their percentage of the total power (LF power\% and HF power\%), the power in the LF band normalized to the sum of the LF and HF power (LF power nu) and the values of the frequencies having maximum magnitude (LF peak and HF peak).

\section{Statistical analysis and classification}

To analyze the reliability of LPP in synthetic and experimental series we used the nonparametric Spearman correlation analysis to compare the values of the LPP features calculated in the ultra-short duration of windows with the parameters extracted from the total length of the series [46]. The use of such a non-parametric test is justified by having non-gaussian distribution of the samples $(p<0.05$ of the null hypothesis of having gaussian samples of the Kolmogorov-Smirnov test) [47].

For each parameter and window size, we calculated the percentage absolute error $(\epsilon \%(i, \tau))$ [48] between the mean value of the parameter among all the equal length windows and the value of the parameter estimated in five-minutes series, which is taken as the reference, according to equation 2 :

$$
\epsilon \%(i, \tau)=100 \times\left|\frac{m(i, \tau)-m_{B L}(i)}{m_{B L}(i)}\right|
$$

where $i$ was an integer in the range [1-N], where $\mathrm{N}$ was the total number of the series, $\tau$ was the duration of the selected window, $m(i, \tau)$ was the average metrics (e.g. SD1) calculated from all the windows of the same length, $m_{B L}(i)$ was the reference metrics calculated from the 1-hour synthetic series and from 5-minutes experimental series.

The Bland-Altman plot was analyzed in order to check interchangeability of ultra-short windows and the whole signal in terms of LPP parameters [49], [50]. In the analysis of the values of LPP estimated in the ultra-short time series, we found that there was a raise in the variability of the differences as the magnitude of measurements increased. For this reason, we applied the traditional Bland-Altman plot method to the logarithmic transformations of both measurements, as suggested by the literature [50].

We used the features extracted from the experimental data

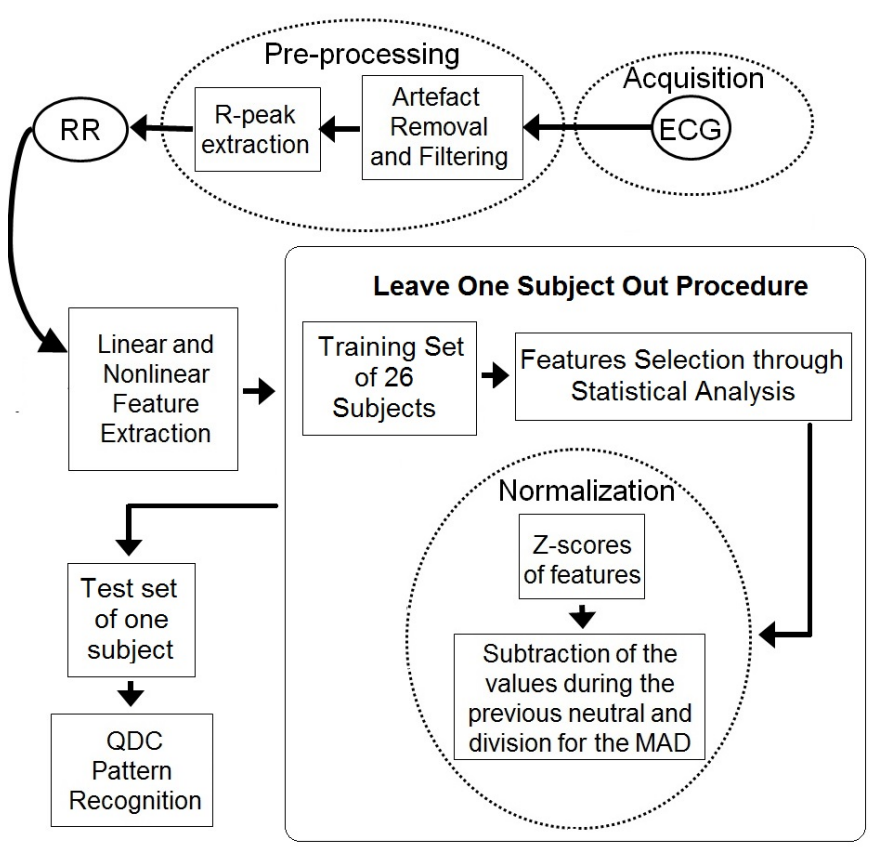

Figure 1. Overall block scheme of the proposed emotion recognition system.

to distinguish the sessions related to negative sounds from the sessions corresponding to the positive stimulation. We implemented a Leave-One-Subject-Out (LOSO) procedure, following the block diagram summarized in Fig.1, where each iteration implied a feature selection and a normalization of the 
Table II

SPEARMAN'S CORRELATION COEFFICIENTS (CALCULATED BETWEEN THE VALUES OF LPP PARAMETERS CALCULATED IN THE TOTAL LENGTH OF THE SERIES AND WITH A WINDOWING OF 15, 35 AND 60 SAMPLES, RESPECTIVELY) AND THE MEDIAN OF PERCENTAGE ABSOLUTE ERRORS. THE VALUES ARE REPORTED FOR ALL THE LAG $M$ OF THE LPP AND FOR THE CORRELATION TESTS THERE WAS A $p-v a l u e<0.00001$.

\begin{tabular}{|c|c|c|c|c|c|c|c|c|c|c|c|c|c|c|c|c|}
\hline \multirow[b]{2}{*}{ M } & & \multicolumn{3}{|c|}{ SD1 } & \multicolumn{3}{|c|}{ SD2 } & \multicolumn{3}{|c|}{ SD12 } & \multicolumn{3}{|c|}{$\mathbf{S}$} & \multicolumn{3}{|c|}{ SDRR } \\
\hline & & 15 sam & 35 sam & 60 sam & 15 sam & 35 sam & $60 \mathrm{sam}$ & 15 sam & 35 sam & $60 \mathrm{sam}$ & $15 \mathrm{sam}$ & 35 sam & 60 sam & 15 sam & 35 sam & 60 sam \\
\hline & $\rho$ & 0,9979 & 0,9983 & 0,9983 & 0,9965 & 0,9987 & 0,9994 & 0,9710 & 0,9905 & 0,9944 & 0,9983 & 0,9992 & 0,9995 & 0,9978 & 0,9992 & 0,9996 \\
\hline 1 & $\epsilon \%$ & 3,27 & 3,27 & 2,97 & 5,66 & 3,65 & 2,69 & 6,92 & 4,16 & 3,13 & 3,28 & 2,07 & 1,30 & 4,68 & 3,36 & 2,57 \\
\hline & $\rho$ & 0,9988 & 0,9993 & 0,9993 & 0,9879 & 0,9974 & 0,9991 & 0,9878 & 0,9960 & 0,9974 & 0,9980 & 0,9992 & 0,9996 & 0,9974 & 0,9992 & 0,99966 \\
\hline 2 & $\epsilon \%$ & 3,22 & 2,88 & 2,41 & 7,11 & 4,13 & 2,92 & 11,86 & 4,67 & 2,94 & 2,99 & 1,90 & 1,26 & 4,65 & 3,39 & 2,59 \\
\hline & $\rho$ & 0,9987 & 0,9995 & 0,9997 & 0,9938 & 0,9991 & 0,9996 & 0,9736 & 0,9919 & 0,9944 & 0,9961 & 0,9993 & 0,9996 & 0,9964 & 0,9992 & 0,9996 \\
\hline 3 & $\epsilon \%$ & 4,60 & 3,44 & 2,67 & 7,27 & 4,37 & 3,15 & 8,83 & 3,36 & 2,29 & 3,03 & 1,52 & 0,99 & 4,62 & 3,41 & 2,62 \\
\hline & $\rho$ & 0,9973 & 0,9992 & 0,9996 & 0,9923 & 0,9991 & 0,9994 & 0,9 & 0,9841 & 0,9862 & 0,9962 & 0,9995 & 0,9997 & 0,9945 & 0,9992 & 0,9996 \\
\hline 4 & $\epsilon \%$ & 4,63 & 3,34 & 2,59 & 7,99 & 4,72 & 3,23 & 12,63 & 3,57 & 2,0 & 5,3 & 2,05 & 1,28 & 4, & 3,43 & 2,65 \\
\hline & $\rho$ & 0,9924 & 0,9983 & 0,9991 & 0,9690 & 0,9979 & 0,9988 & 0,9856 & 0,9931 & 0,9943 & 0,9893 & 0,9987 & 0,9992 & 0,9911 & 0,9992 & 0,99966 \\
\hline 5 & $\epsilon \%$ & 6,33 & 4,12 & 3,01 & 7,98 & 4,96 & 3,4 & 16 & 5,33 & 2,9 & & 4,41 & 20 & & 3,44 & 2,66 \\
\hline & & 0,9796 & 0,9987 & 0,9994 & 0,9808 & 0,9982 & & & 0,9904 & & & 0,9985 & & & 0,9991 & 0,9996 \\
\hline 6 & $\epsilon \%$ & 6,85 & 4.31 & 3,18 & 6,88 & 4,60 & 3,5 & 10,90 & 3,92 & 2,67 & 8,30 & 3,92 & 2,75 & 4, & 3,49 & 2,66 \\
\hline & $\rho$ & 0,9651 & 0,9985 & 0,9993 & 0,9828 & 0,9982 & & & 0,9939 & & & 0,9977 & & & 991 & 0,9996 \\
\hline 7 & $\epsilon \%$ & 7,75 & 3,90 & 2,95 & 7,45 & 4,95 & 3,3 & 15 , & 5,25 & 2,77 & 7,4 & 3,39 & 2,1 & 5 & 3,51 & 2,65 \\
\hline & & 0,9755 & 0,9990 & 0,9993 & & 0,9986 & & & 0,9954 & & & 0,9983 & & & 0,9991 & 0,9996 \\
\hline 8 & $\epsilon \%$ & 11,67 & 3,70 & 2,81 & 9,4 & 5,07 & & & 6,30 & & & 3,99 & & & 3,49 & 2,68 \\
\hline & & 0,9481 & 0,9988 & 0,9994 & 0,9 & 0,9988 & & & 0,9916 & & & 0,9986 & 94 & & 0,9990 & 0,9996 \\
\hline 9 & $\epsilon \%$ & 16,19 & 3,36 & 2,52 & 11,61 & 5,04 & 3,7 & 15 & 7,32 & 4,8 & 23 & 3,2 & & 10 & 3, & 2,69 \\
\hline & $\rho$ & 0,8860 & 0,9989 & 0,9994 & 0,9677 & 0,9987 & 0,9993 & 0,9290 & 0,9947 & 0,9966 & 0,9274 & 0,9984 & 0,9992 & 0,9417 & 0,9990 & 0,9996 \\
\hline 10 & $\epsilon \%$ & 20,97 & 3,44 & 2,44 & 18,75 & 5,11 & 3,75 & 25,50 & 8,67 & 5,51 & 32,71 & 3,59 & 2,01 & 14,82 & 3,47 & 2,70 \\
\hline
\end{tabular}

training set on $\mathrm{N}-1$ subjects. The feature selection was made taking into consideration the ten parameters which presented the lowest p-values obtained by Wilcoxon non-parametric statistical test between the two valence levels [51]. Before performing the statistical analysis, Kolmogorov-Smirnov tests were applied in order to check that the data were not normally distributed. For each of the LOSO steps, the training set was normalized by means of the non-parametric version of $\mathrm{z}$ score approach. The median and median absolute deviation (MAD) values of the training set (all the arousal sessions over $\mathrm{N}-1$ subjects) were used to normalize the test sample according to the z-score definition. In the normalization of the dataset we implemented two steps: each feature value extracted from each subject during the neutral session was subtracted from the corresponding value estimated during the successive negative or positive valence session; the value obtained from the previous step was divided by the MAD evaluated over all the values of that parameter of all the subjects involved in the training set for the considered valence session.

In the LOSO procedure only features extracted from positive and negative valence sessions were taken in consideration, so we considered a total of 208 samples in the training set (four values for the negative valence and four values for the positive valence for each of 26 subjects), and 8 samples for the test set. The classifier employed in this study was a Quadratic Discriminant Classifier (QDC), which is based on the Bayesan decision theory [52], [53].

\section{EXPERIMENTAL RESULTS}

\section{A. Reliability of LPP parameters from synthetic data}

The results of the Spearman's rank correlation tests highlighted a very good correlation between the parameters calculated in the ultra-short windows and the features belonging to the baseline.

The results of the correlation coefficients $\rho$ are reported in Table II, for the durations of the windows of 15 (i.e. the shortest window considered in the study), 35 and 60 samples (i.e. two examples of ultra-short lengths, including the length selected to study the experimental data). The $p$-values of the test correlations were less than $10^{-5}$ in all cases of study. We applied this test for all values of LPP parameters in all $M$ lags, from 1 to 10 and for all considered lengths. As we can see in Table II the condition $\rho>0.90$ is always fulfilled, except for the comparison between the SD1 value obtained for the windows of 15 samples at $10^{\text {th }}$ lag, i.e. taking in consideration only five samples in the scatterplot. Specifically for the durations of 35 samples and 60 samples the correlation coefficients are always above 0.99 , indicating a very good agreement between the two measurements and the real values. Concerning the evaluation of the percentage absolute error $\epsilon \%$, the LPP parameter which showed the lowest divergence when it was calculated in ultra-short widows was $S$, the area of the imaginary ellipse drawn on the points of the PP. Figure 2 illustrates the trend of the median of the $\epsilon \%_{S}$ for all of the window sizes from 15 samples to 300 samples (about 5 minutes, i.e. the standard duration for short term HRV analysis), considering the first lag. For this LPP parameter the median of the values of $\epsilon \%$ resulted to be in the range between $1.01 \%$ (with 130 samples) and $3.28 \%$ (with 15 samples). This error of $3.28 \%$ went up to $32.71 \%$ when we considered

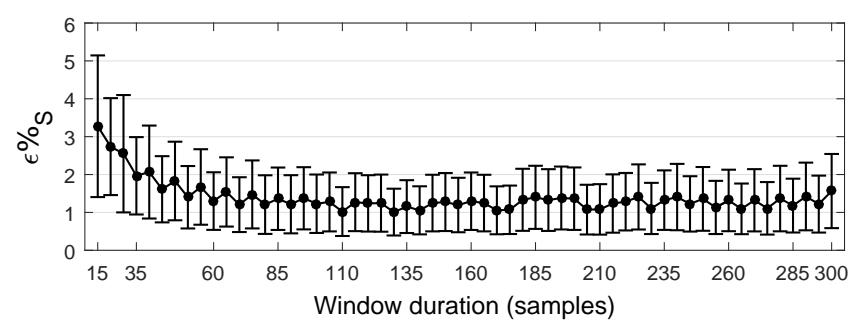

Figure 2. Percent difference calculated from the values of $\mathrm{S}$ parameter in ultra-short series in comparison with the values in the total series. The errors are shown as median and MAD of $\epsilon \%_{S}$.

the tenth lag for the parameter $\mathrm{S}$ in series of 15 samples. This condition indicated that even if this length allowed to 
calculate LPP features highly correlated with those extracted from the total duration of the signals, these parameters had a strong systematic error when the lag increased. This error was reduced when the number of samples raised. In Figure 3 the
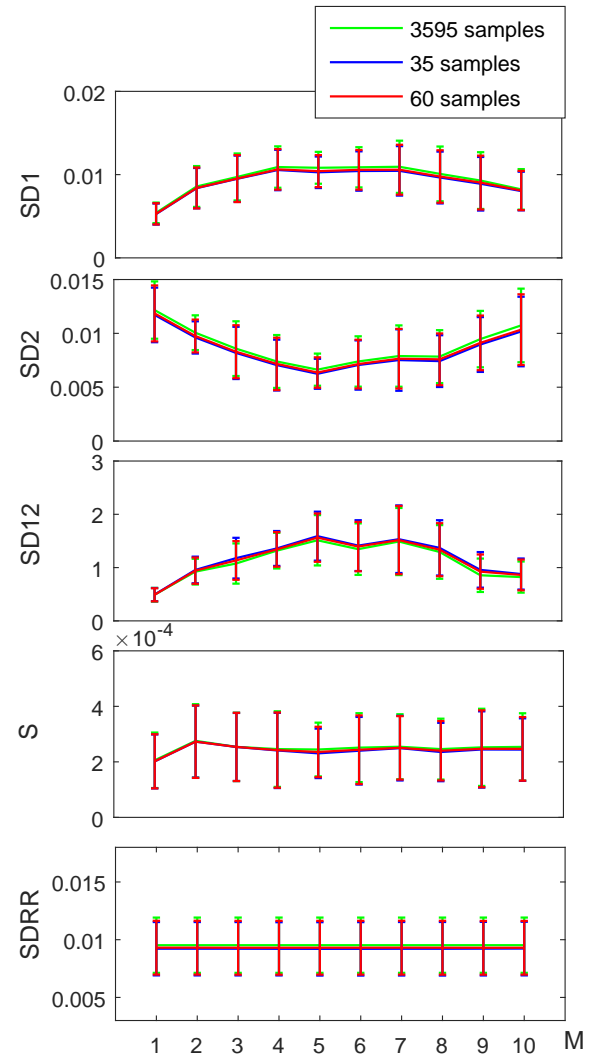

Figure 3. LPP parameters as function of $M$ lag. The value of the parameters are presented as median and MAD calculated on the 1200 synthetic series, for three window size: 35 samples (blue), 60 samples (red) and 3595 samples, the total length of series (green).

median values of the five LPP parameters and related MAD are shown as a function of the lag M. It is easily observable that when we compared the values in two different ultra-short windows (35 samples and 60 samples) with the same features extracted in the total signals, the trends of the parameters are nearly overlapped.

In Figures 4, 5 we show the Bland-Altman plots for the logtransformed data of the parameters SD1 and S, comparing the values in three ultra-short lengths of the series (15 samples, 35 samples and 60 samples) and the values for the total duration of the synthetic signals. In each Bland-Altman plot the median values of the differences between LPP parameters in the two considered lengths of the windows are reported, joined to the limits of agreement. The limits of agreement used in these graphs are calculated as the $1.96 \times 1.4826 \times M A D$, which indicates the $95 \%$ interval of confidence for non-gaussian variables. As we can see in these plots, most of the differences lie between the limits of agreement, especially when we look at the graphs constructed with the LPP values of 35 and 60 samples.
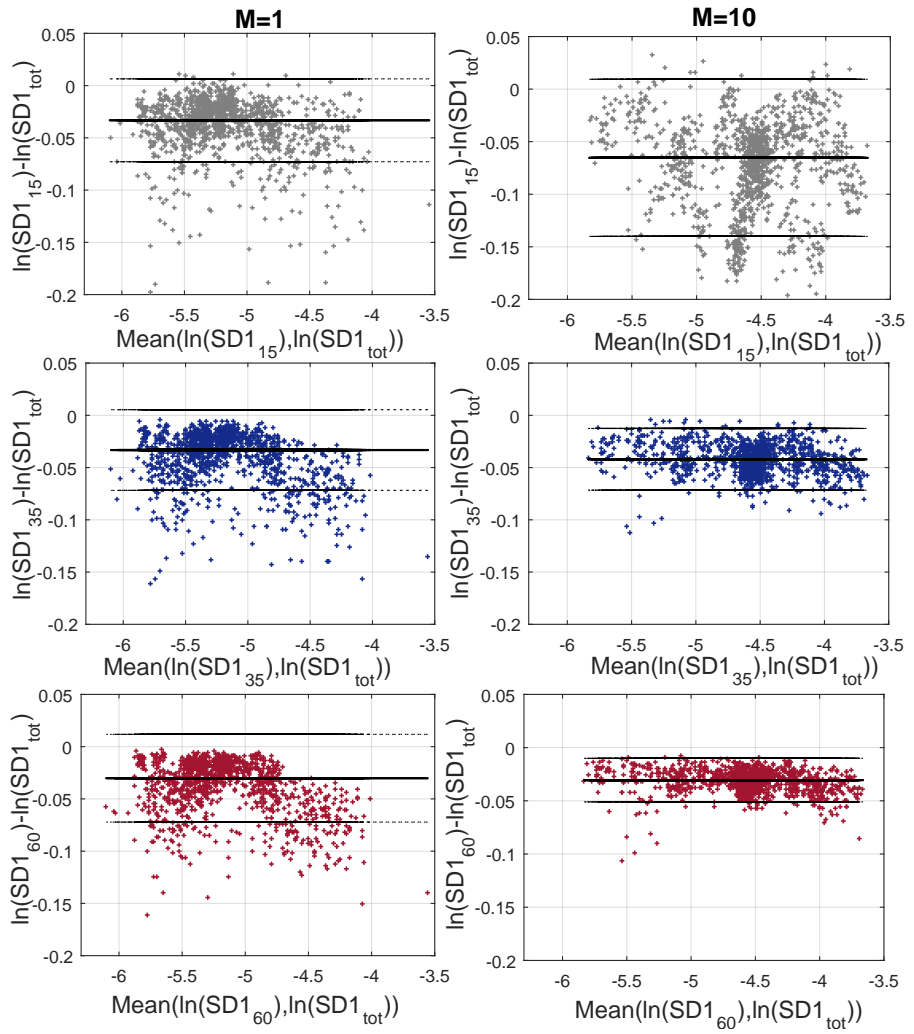

Figure 4. Bland-Altman plots of agreement for SD1 derived by the 15 samples (gray, on the top), 35 samples (blue, in the middle) and 60 samples (red, on the bottom) windows and SD1 extracted from the total length of synthetic series. The plots on the left are referred to the first lag of LPP and the plots on the right regard the last lag.

\section{B. Application to experimental data}

Table III shows the statistical results obtained from the application of Spearman correlation test to real data acquired during resting state, comparing the LPP parameters extracted from 15, 35 and 60 seconds windows and from the whole segments of 5-minutes. The Spearman correlation coefficient was always higher than 0.9 for the parameters SD1 and S, also considering 15 seconds windows. For SDRR $\rho$ went below 0.9 when it was computed in 15 seconds windows with a lag above six. SD2 presented a correlation coefficient always higher than 0.7 , whereas for SD12 the values of $\rho$ decreased especially in higher lags when 15 seconds were examined.

With reference to the computation of the percentage absolute error, the lowest divergence was found in the values of SD1. Considering the first lag, the values of $\epsilon \%$ (SD1) reached a minimum of $0.62 \%$ with 35 seconds windows and a maximum of $4.14 \%$ with 150 seconds.

The LPP parameters were extracted from ultra-short time windows, along with standard HRV features, from RR series recorded during an emotional stimulation conducted on 27 subjects through IADS database. The purpose of the study was to verify the reliability of the parameters derived from ultra-short series, to discern the valence dimension of the stimuli.

We applied the algorithm described in Section II-B2 and in 
Table III

SPEARMAN'S CORRELATION COEFFICIENTS (CALCULATED BETWEEN THE VALUES OF LPP PARAMETERS CALCULATED IN 5-MINUTES EXPERIMENTAL SERIES AND WITH A WINDOWING OF 15, 35 AND 60 SECONDS, RESPECTIVELY) AND THE MEDIAN OF PERCENTAGE ABSOLUTE ERRORS. THE VALUES ARE REPORTED FOR ALL THE LAG $M$ OF THE LPP AND THERE WAS A $p-$ value $<0.00001$ FOR THE CORRELATION TESTS APPLIED TO SD1, SD2, S AND SDRR, WHEREAS $p<0.05$ FOR SD12.

\begin{tabular}{|c|c|c|c|c|c|c|c|c|c|c|c|c|c|c|c|c|}
\hline \multirow[b]{2}{*}{ M } & & \multicolumn{3}{|c|}{ SD1 } & \multicolumn{3}{|c|}{ SD2 } & \multicolumn{3}{|c|}{ SD12 } & \multicolumn{3}{|c|}{$\mathbf{S}$} & \multicolumn{3}{|c|}{ SDRR } \\
\hline & & $15 \mathrm{sec}$ & $35 \mathrm{sec}$ & $60 \mathrm{sec}$ & $15 \mathrm{sec}$ & $35 \mathrm{sec}$ & $60 \mathrm{sec}$ & $15 \mathrm{sec}$ & $35 \mathrm{sec}$ & $60 \mathrm{sec}$ & $15 \mathrm{sec}$ & $35 \mathrm{sec}$ & $60 \mathrm{sec}$ & $15 \mathrm{sec}$ & $35 \mathrm{sec}$ & $60 \mathrm{sec}$ \\
\hline \multirow{2}{*}{1} & $\rho$ & 0,9993 & 0,9993 & 0,9967 & 0,8486 & 0,9036 & 0,9079 & 0,8812 & 0,9373 & 0,9608 & 0,9758 & 0,9879 & 0,9894 & 0,9018 & 0,9267 & 0,9436 \\
\hline & $\epsilon \%$ & 1,47 & 0,62 & 2,02 & 24,43 & 15,82 & 10,18 & 39,59 & 21,84 & 14,47 & 20,86 & 14,04 & 9,97 & 20,05 & 12,97 & 8,60 \\
\hline \multirow{2}{*}{2} & $\rho$ & 0,9974 & 0,9989 & 0,9945 & 0,8061 & 0,8471 & 0,8812 & 0,7313 & 0,8622 & 0,9201 & 0,9670 & 0,9765 & 0,9806 & 0,9065 & 0,9150 & 0,9414 \\
\hline & $\epsilon \%$ & 1,31 & 1,04 & 1,88 & 31,72 & 18,52 & 13,38 & 57,59 & 28,54 & 18,03 & 27,66 & 16,97 & 12,79 & 20,15 & 13,23 & 9,15 \\
\hline \multirow{2}{*}{3} & $\rho$ & 0,9912 & 0,9974 & 0,9978 & 0,7518 & 0,8288 & 0,8735 & 0,7768 & 0,9032 & 0,9120 & 0,9637 & 0,9762 & 0,9806 & 0,9091 & 0,9194 & 0,9391 \\
\hline & $\epsilon \%$ & 3,07 & 1,76 & 2,15 & 30,87 & 19,80 & 13,32 & 54,00 & 28,03 & 18,32 & 28,06 & 19,25 & 13,53 & 20,18 & 13,34 & 9,20 \\
\hline \multirow{2}{*}{4} & $\rho$ & 0,9857 & 0,9831 & 0,9736 & 0,7108 & 0,7870 & 0,8875 & 0,7812 & 0,8684 & 0,9109 & 0,9436 & 0,9685 & 0,9824 & 0,8988 & 0,9076 & 0,9384 \\
\hline & $\epsilon \%$ & 5,02 & 2,20 & 2,72 & 31,90 & 20,14 & 14,43 & 45,54 & 24,91 & 14,89 & 29,87 & 20,19 & 13,60 & 20,60 & 13,66 & 9,08 \\
\hline \multirow{2}{*}{5} & $\rho$ & 0,9879 & 0,9930 & 0,9934 & 0,7089 & 0,7764 & 0,8090 & 0,4333 & 0,5971 & 0,7595 & 0,9300 & 0,9626 & 0,9758 & 0,9003 & 0,9098 & 0,9373 \\
\hline & $\epsilon \%$ & 5,95 & 1,30 & 3,06 & 37,91 & 23,25 & 16,19 & 61,84 & 30,88 & 20,77 & 36,40 & 21,07 & 14,92 & 21,21 & 13,71 & 9,13 \\
\hline \multirow{2}{*}{6} & $\rho$ & 0,9890 & 0,9949 & 0,9864 & 0,7955 & 0,8365 & 0,8827 & 0,2621 & 0,4648 & 0,6133 & 0,9384 & 0,9611 & 0,9718 & 0,9058 & 0,9164 & 0,9410 \\
\hline & $\epsilon \%$ & 6,39 & 1,89 & 3,07 & 39,04 & 26,36 & 17,86 & 66,67 & 40,93 & 24,99 & 39,98 & 24,41 & 17,11 & 21,40 & 14,19 & 9,41 \\
\hline \multirow{2}{*}{7} & $\rho$ & 0,9853 & 0,9960 & 0,9923 & 0,7969 & 0,8537 & 0,8845 & 0,5854 & 0,6947 & 0,7500 & 0,9443 & 0,9630 & 0,9813 & 0,8919 & 0,9142 & 0,9402 \\
\hline & $\epsilon \%$ & 8,95 & 2,53 & 2,92 & 35,98 & 24,88 & 17,67 & 50,12 & 33,54 & 20,61 & 39,77 & 23,98 & 17,51 & 21,40 & 14,57 & 9,30 \\
\hline \multirow{2}{*}{8} & $\rho$ & 0,9861 & 0,9938 & 0,9894 & 0,8402 & 0,8944 & 0,9311 & 0,5931 & 0,6829 & 0,7471 & 0,9351 & 0,9630 & 0,9773 & 0,8886 & 0,9135 & 0,9413 \\
\hline & $\epsilon \%$ & 11,24 & 2,23 & 3,08 & 36,38 & 25,20 & 16,66 & 48,53 & 31,68 & 17,65 & 41,26 & 23,93 & 15,73 & 21,80 & 14,72 & 9,17 \\
\hline \multirow{2}{*}{9} & $\rho$ & 0,9707 & 0,9897 & 0,9886 & 0,8354 & 0,8930 & 0,9117 & 0,3296 & 0,6096 & 0,7922 & 0,9403 & 0,9648 & 0,9765 & 0,8889 & 0,9139 & 0,9413 \\
\hline & $\epsilon \%$ & 12,26 & 2,64 & 3,16 & 32,94 & 25,08 & 15,97 & 41,42 & 31,07 & 19,69 & 42,17 & 23,91 & 15,52 & 23,22 & 14,79 & 9,27 \\
\hline \multirow{2}{*}{10} & $\rho$ & 0,9516 & 0,9883 & 0,9798 & 0,8310 & 0,8827 & 0,9109 & 0,3240 & 0,6056 & 0,7214 & 0,9443 & 0,9688 & 0,9802 & 0,9003 & 0,9244 & 0,9421 \\
\hline & $\epsilon \%$ & 16,62 & 3,57 & 2,89 & 32,20 & 22,55 & 15,53 & 36,09 & 29,33 & 19,12 & 43,04 & 23,70 & 14,24 & 25,21 & 15,06 & 9,58 \\
\hline
\end{tabular}
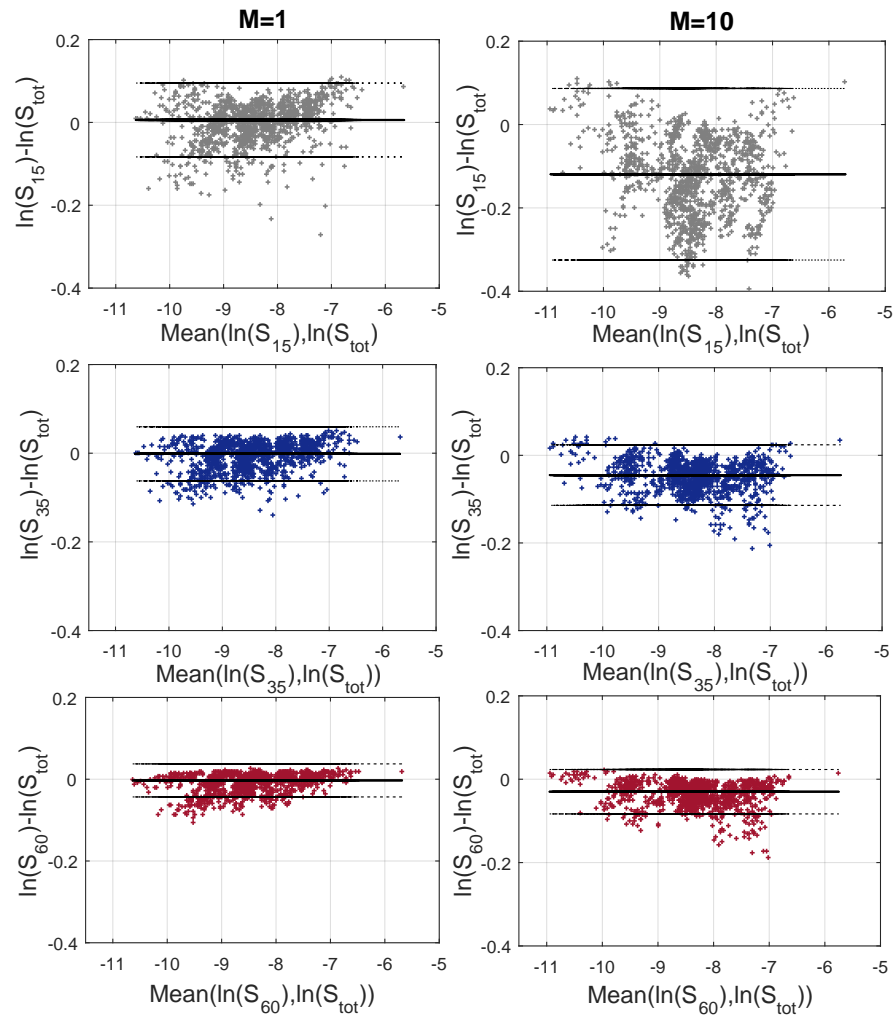

Figure 5. Bland-Altman plots of agreement for S derived by the 15 samples (gray, on the top), 35 samples (blue, in the middle) and 60 samples (red, on the bottom) windows and $\mathrm{S}$ extracted from the total length of synthetic series. The plots on the left are referred to the first lag of LPP and the plots on the right regard the last lag.

Fig.1 in the two cases of study, with time windows of 1 minute and 28 seconds and time windows of 35 seconds.

In Table IV the ten most selected features through the statistical analysis tests between the two levels of valence are shown, in the case of 35 seconds windowing. In the case of LPP parameters the increase or decrease of the median values are presented, going from the session of negative valence to the session of positive sounds.

Table IV

THE TEN MOST SELECTED FEATURES IN THE LOSO PROCEDURE THROUGH WILCOXON TEST, APPLIED TO THE PARAMETERS EXTRACTED

FROM 35 SECONDS SEGMENTS. THE SYMBOL (-) HIGHLIGHTS A DECREASE OF THE RELATED LPP PARAMETER IN ACCORDANCE WITH THE INCREASE OF THE VALENCE, WHEREAS THE SYMBOL (+) INDICATES AN INCREASE (CONSIDERING THE MEDIAN VALUES ON THE 27 SUBJECTS). CONCERNING THE NOTATION USED IN THIS TABLE, THE SUBSCRIPTS INDICATE THE LAG VALUE (E.G. SD $1_{7}$ SYMBOLIZES SD1 PARAMETER COMPUTED AT M=7).

\begin{tabular}{|c|c|c|}
\hline Standard Features & Nonlinear features & All features \\
\hline $\mathrm{LF} / \mathrm{HF}$ & SD1210 (-) & $\mathrm{RR}$ std \\
\hline LF power nu & $\mathrm{SD}_{6}{ }_{(-)}$ & SD12 $10(-)$ \\
\hline LF power \% & $\mathrm{SD}_{5}(-)$ & $\mathrm{SD}_{6}(-)$ \\
\hline LF power & $\mathrm{SD}_{7}(+)$ & $\mathrm{SD}_{5}(-)$ \\
\hline TIRR & $\mathrm{SDRR}_{7}(-)$ & TIRR \\
\hline RR std & $\mathrm{SD}_{4}(+)$ & $\mathrm{SD}_{7}(+)$ \\
\hline HF peak & $\mathrm{SD} 1_{10}(-)$ & $\mathrm{SD}_{4}(+)$ \\
\hline RMSSD & $\mathrm{SDRR}_{5}(-)$ & LF power $\%$ \\
\hline HF power \% & $\mathrm{SDRR}_{8}(-)$ & $\mathrm{LF} / \mathrm{HF}$ \\
\hline RR mean & SD125 (-) & $\mathrm{SD}_{2} 2_{5}(-)$ \\
\hline
\end{tabular}

Table $\mathrm{V}$ shows the confusion matrices related to the results of recognition algorithm applied using three different datasets: only standard HRV features, only nonlinear LPP parameters and all the features together. For each dataset both window durations were considered. Concerning the classification made with only the standard HRV features (time and frequency domain), the accuracies of the classification were $65.74 \%$ and $68.06 \%$ respectively. The best outcomes in valence recognition were reached using the LPP parameters and the values of accuracy went up to $77.78 \%$ and $79.17 \%$ respectively.

Table $\mathrm{V}$ also reports the confusion matrices obtained while discerning the valence dimension of the acoustic emotional stimulation, using all the features as input of the algorithm. In that case, the accuracies of the algorithm for valence level 
Table V

CONFUSION MATRICES OF QDC ClassifiER FOR VALENCE LEVEL RECOGNITION, USING THREE DIFFERENT DATASETS (ONLY STANDARD FEATURES, ONLY NONLINEAR AND ALL THE FEATURES TOGETHER) AND TWO DIFFERENT WINDOW LENGTHS (1 MINUTE AND 28 SECONDS AND 35 SECONDS).

\begin{tabular}{|c|c|c|c|c|}
\hline Dataset & Window duration & & nfusion I & trix \\
\hline \multirow{6}{*}{ Standard } & \multirow{3}{*}{$1 ' 28^{\prime \prime}$} & & NEG & POS \\
\hline & & NEG & 74.0741 & 42.5926 \\
\hline & & POS & 25.9259 & 57.4074 \\
\hline & \multirow{3}{*}{$35^{\prime \prime}$} & & NEG & POS \\
\hline & & NEG & 66.6667 & 30.5556 \\
\hline & & POS & 33.3333 & 69.4444 \\
\hline \multirow{6}{*}{ Non-linear } & \multirow{3}{*}{$1 ' 28$ ' } & & NEG & POS \\
\hline & & NEG & 68.5185 & 12.9630 \\
\hline & & POS & 31.4815 & 87.0370 \\
\hline & \multirow{3}{*}{$35^{\prime \prime}$} & & NEG & POS \\
\hline & & NEG & 69.4444 & 11.1111 \\
\hline & & POS & 30.5556 & 88.8889 \\
\hline \multirow{6}{*}{$\begin{array}{c}\text { Standard } \\
+ \\
\text { Non-linear }\end{array}$} & \multirow{3}{*}{$1 ' 28$ ' } & & NEG & POS \\
\hline & & NEG & 65.7407 & 12.0370 \\
\hline & & POS & 34.2593 & 87.9630 \\
\hline & \multirow{3}{*}{$35^{\prime \prime}$} & & NEG & POS \\
\hline & & NEG & 68.5185 & 17.5926 \\
\hline & & POS & 31.4815 & 82.4074 \\
\hline
\end{tabular}

recognition were $76.85 \%$ (using 1 minute and 28 seconds) and $75.46 \%$ (for 35 seconds windows).

\section{DISCUSSION}

In this work we presented a study on the reliability of LPP parameters in ultra-short term analysis. We analyzed the behavior of these features using synthetic series, in order to have results which were not biased by the experimental condition and then, we tested the parameters on experimental ECG data recorded on 32 healthy subjects, during 5-minutes resting state. LPP practice is a complete description of the autocovariance function and of power spectrum of the intervals [54]. However, the properties of LPP had not yet been investigated in relation with the duration of the time window. The use of two different datasets (synthetic and real series) allowed a complete overview of the validation of the method. In this paper we showed a very good reliability of the LPP parameters for ultra-short series in 1200 synthetic series generated with the IPFM model, through the analysis of Spearman's rank correlation, relative errors values and BlandAltman plot. The correlation coefficients, calculated between the LPP parameters extracted in ultra-short series and in the total length of the signals ( 1 hour), were in almost all the cases higher than 0.9 for all of the lags.

Nonlinear parameters have been not recommended by the literature, given the low reliability demonstrated in some previous studies. McNames et al. for example found a relative error of about $100 \%$ for the calculation of ApEn in series with a duration less than 40 seconds [23]. We demonstrated through the use of synthetic data, which allows to guarantee that the signal characteristics do not change within the whole time interval, that LPP parameters conserve their effectiveness also in series which last less than one minute.

Considering that in the literature about reliability of HRV parameters, the features supposed to be the most useful for series shorter than 5 minutes were those belonging to the time domain [17], [20], [21], we expected that the PP technique outperformed the methods obtained by means of nonlinear theory, given its acknowledged relation for example with the standard deviation of the RR intervals [54].

The reliability of LPP approach in ultra-short RR series was confirmed in the analysis of real data. In this case SD1 was found to be the parameter with the lowest percentage absolute error, whereas SD2 presented an higher divergence in comparison with the synthetic series results. Real-data can be affected by slow non-stationatities and trends that can increase the error of SD2, which is more correlated with lowfrequency oscillations in HRV, as suggested in the literature [54]. Studying the usefulness of LPP method in real data we also experimented its application to 10 seconds series, which is the classical resting ECG test duration. We analyzed five values of lag and the results were very promising especially for SD1 parameters, with a percentage error below $5 \%$ in the first three lags. The Spearman correlation coefficient was higher than 0.89 for SD1, SDRR and S parameters, considering lags from 1 to 5 .

Previous studies suggested that LPP method has a relevant impact in the study of acoustic stimulation protocols [42], [55]-[57], so we tested its reliability also using experimental RR series collected from 27 healthy subjects, during acoustic stimulation. We applied an ad-hoc algorithm studied to compute a LOSO procedure and we reached a significant accuracy of recognition of the pleasantness/unpleasantness sessions of the experimental protocol, using two lengths of time windows: 1 minute and 28 seconds and 35 seconds. The first length was the length of the neutral session in the protocol (used to normalize the dataset) and the second was chosen because we considered that a frequency resolution of $\delta f=1 / 35 \mathrm{~s}$ $=0,029 \mathrm{~Hz}$, can be sufficient to reliably estimate all HRV measures. Indeed, we were interested not only in studying the performance of LPP parameters in discriminating positive and negative valence auditory stimuli, but also in compare their performance with linear indexes, so we needed a time window long enough to estimate reliably these parameters. In spite of one of our previous studies [42], where only windows of 1 minute and 28 seconds were analyzed, in this study we also included the analysis of 35 seconds series. Moreover here we disregarded the features needing a higher number of samples, such as Very Low Frequency (VLF) parameters, Detrended Fluctuation Analysis (DFA) and ApEn, restricting the non-linear analysis to the LPP parameters. We calculate the accuracy of the recognition algorithm in three cases: using standard and nonlinear features, with only the standard parameters and with only the LPP parameters. The highest accuracy was obtained considering only the nonlinear features as input of the LOSO procedure, reaching the values of $77.78 \%$ and $79.17 \%$ for the two lengths of window. Further, in the LOSO procedure, most of the features selected using statistical between the levels of the valence, were the LPP features (see Table IV), when all the dataset was considered. This findings also confirm the efficiency of LPP method in studies involving emotion recognition [16] and particular 
psico-behavioral state, i.e.meditation [25]. The decrease in the median values of the LPP parameters in accordance with the increase of the valence from negative to positive sounds, implies significant changes in the ANS dynamical response to standardized sounds. SD1 and SD12 parameters have been described in previous works as indexes of parasympathetic activity [58], [59] and these findings show its progressive decline during the arousing stimulation.

In this work we have studied the reliability of LPP parameters extracted from ultra-short time RR series both in simulated and real data. Results on simulation data showed that LPP parameters derived from RR series longer than $35 \mathrm{~s}$ can be interchanged with those derived from $1 \mathrm{~h}$ with high Spearman correlation and low error. Results on real data show that LPP parameters can discriminate positive and negative valence acoustic stimuli with an accuracy of $79.17 \%$, outperforming standard time and frequency HRV parameters. These results support the use of LPP parameters in ultra-short HRV analysis for emotion recognition.

\section{REFERENCES}

[1] T. F. of the European Society of Cardiology, T. F. of the European Society of Cardiology et al., "the north american society of pacing and electrophysiology. heart rate variability: standards of measurement, physiological interpretation and clinical use," Circulation, vol. 93, no. 5, pp. 1043-1065, 1996.

[2] R. Sassi, S. Cerutti, F. Lombardi, M. Malik, H. V. Huikuri, C.-K. Peng, G. Schmidt, Y. Yamamoto, B. Gorenek, G. H. Lip et al., "Advances in heart rate variability signal analysis: joint position statement by the ecardiology esc working group and the european heart rhythm association co-endorsed by the asia pacific heart rhythm society," Europace, p. euv015, 2015.

[3] R. E. Kleiger, J. P. Miller, J. T. Bigger, and A. J. Moss, "Decreased heart rate variability and its association with increased mortality after acute myocardial infarction," The American journal of cardiology, vol. 59, no. 4, pp. 256-262, 1987.

[4] R. M. Carney, J. A. Blumenthal, P. K. Stein, L. Watkins, D. Catellier, L. F. Berkman, S. M. Czajkowski, C. OâĂŹConnor, P. H. Stone, and K. E. Freedland, "Depression, heart rate variability, and acute myocardial infarction," Circulation, vol. 104, no. 17, pp. 2024-2028, 2001.

[5] R. Furlan, S. Guzzetti, W. Crivellaro, S. Dassi, M. Tinelli, G. Baselli, S. Cerutti, F. Lombardi, M. Pagani, and A. Malliani, "Continuous 24hour assessment of the neural regulation of systemic arterial pressure and $\mathrm{rr}$ variabilities in ambulant subjects." Circulation, vol. 81, no. 2, pp. 537-547, 1990.

[6] H. Mølgaard, K. E. Sørensen, and P. Bjerregaard, "Attenuated 24-h heart rate variability in apparently healthy subjects, subsequently suffering sudden cardiac death," Clinical Autonomic Research, vol. 1, no. 3, pp. 233-237, 1991.

[7] G. Speranza, G. Nollo, F. Ravelli, and R. Antolini, "Beat-to-beat measurement and analysis of the $\mathrm{rt}$ interval in $24 \mathrm{~h}$ ecg holter recordings," Medical and Biological Engineering and Computing, vol. 31, no. 5, pp. 487-494, 1993.

[8] J. E. Hartikainen, K. U. Tahvanainen, and T. A. Kuusela, "Short-term measurement of heart rate variability," in Clinical guide to cardiac autonomic tests. Springer, 1998, pp. 149-176.

[9] G. R. Sandercock, R. Grocott-Mason, and D. A. Brodie, "Changes in short-term measures of heart rate variability after eight weeks of cardiac rehabilitation," Clinical Autonomic Research, vol. 17, no. 1, pp. 39-45, 2007.

[10] R. Steeds, J. Fletcher, M. Smith, J. West, K. Channer, and J. Townend, "Prognostic significance of early short-term measurements of heart rate variability following acute myocardial infarction," The American journal of cardiology, vol. 94, no. 10, pp. 1275-1278, 2004.

[11] L. Salahuddin, J. Cho, M. G. Jeong, and D. Kim, "Ultra short term analysis of heart rate variability for monitoring mental stress in mobile settings," in 2007 29th Annual International Conference of the IEEE Engineering in Medicine and Biology Society. IEEE, 2007, pp. 46564659.
[12] L. Salahuddin, M. G. Jeong, and D. Kim, "Ultra short term analysis of heart rate variability using normal sinus rhythm and atrial fibrillation ecg data," in 2007 9th International Conference on e-Health Networking, Application and Services. IEEE, 2007, pp. 240-243.

[13] S. Boonnithi and S. Phongsuphap, "Comparison of heart rate variability measures for mental stress detection," in 2011 Computing in Cardiology. IEEE, 2011, pp. 85-88.

[14] D. Kim, Y. Seo, S.-h. Kim, and S. Jung, "Short term analysis of long term patterns of heart rate variability in subjects under mental stress," in 2008 International Conference on BioMedical Engineering and Informatics, vol. 2. IEEE, 2008, pp. 487-491.

[15] K. Schaaff and M. T. Adam, "Measuring emotional arousal for online applications: evaluation of ultra-short term heart rate variability measures," in Affective Computing and Intelligent Interaction (ACII), 2013 Humaine Association Conference on. IEEE, 2013, pp. 362-368.

[16] M. Nardelli, G. Valenza, M. Bianchi, A. Greco, A. Lanata, A. Bicchi, and E. P. Scilingo, "Gender-specific velocity recognition of caress-like stimuli through nonlinear analysis of heart rate variability," in 2015 37th Annual International Conference of the IEEE Engineering in Medicine and Biology Society (EMBC). IEEE, 2015, pp. 298-301.

[17] E. Karp, A. Shiyovich, D. Zahger, H. Gilutz, A. Grosbard, and A. Katz, "Ultra-short-term heart rate variability for early risk stratification following acute st-elevation myocardial infarction," Cardiology, vol. 114, no. 4, pp. 275-283, 2009.

[18] S. M. Ostojic, G. Markovic, J. Calleja-Gonzalez, D. Jakovljevic, V. Vucetic, and M. Stojanovic, "Ultra short-term heart rate recovery after maximal exercise in continuous versus intermittent endurance athletes," European journal of applied physiology, vol. 108, no. 5, pp. 1055-1059, 2010.

[19] S. M. Ostojic, M. D. Stojanovic, and J. Calleja-Gonzalez, "Ultra shortterm heart rate recovery after maximal exercise: relations to aerobic power in sportsmen," Chin J Physiol, vol. 54, no. 2, pp. 105-110, 2011.

[20] U. Nussinovitch, K. P. Elishkevitz, K. Katz, M. Nussinovitch, S. Segev, B. Volovitz, and N. Nussinovitch, "Reliability of ultra-short ecg indices for heart rate variability," Annals of Noninvasive Electrocardiology, vol. 16, no. 2, pp. 117-122, 2011.

[21] E. B. Schroeder, E. A. Whitsel, G. W. Evans, R. J. Prineas, L. E. Chambless, and G. Heiss, "Repeatability of heart rate variability measures," Journal of electrocardiology, vol. 37, no. 3, pp. 163-172, 2004.

[22] J. S. Richman and J. R. Moorman, "Physiological time-series analysis using approximate entropy and sample entropy," American Journal of Physiology-Heart and Circulatory Physiology, vol. 278, no. 6, pp. H2039-H2049, 2000.

[23] J. McNames and M. Aboy, "Reliability and accuracy of heart rate variability metrics versus ecg segment duration," Medical and Biological Engineering and Computing, vol. 44, no. 9, pp. 747-756, 2006.

[24] P. Contreras, R. Canetti, and E. R. Migliaro, "Correlations between frequency-domain hrv indices and lagged poincaré plot width in healthy and diabetic subjects," Physiological measurement, vol. 28, no. 1, p. 85, 2006.

[25] A. Goshvarpour, A. Goshvarpour, and S. Rahati, "Analysis of lagged poincare plots in heart rate signals during meditation," Digital Signal Processing, vol. 21, no. 2, pp. 208-214, 2011.

[26] T. P. Thakre and M. L. Smith, "Loss of lag-response curvilinearity of indices of heart rate variability in congestive heart failure," $B M C$ cardiovascular disorders, vol. 6, no. 1, p. 1, 2006.

[27] L. Claudia, I. Oscar, P.-G. Héctor, and V. J. Marco, "Poincaré plot indexes of heart rate variability capture dynamic adaptations after haemodialysis in chronic renal failure patients," Clinical physiology and functional imaging, vol. 23, no. 2, pp. 72-80, 2003.

[28] M. M. Bradley and P. J. Lang, "The international affective digitized sounds (; iads-2): Affective ratings of sounds and instruction manual," University of Florida, Gainesville, FL, Tech. Rep. B-3, 2007.

[29] R. A. Stevenson and T. W. James, "Affective auditory stimuli: Characterization of the international affective digitized sounds (iads) by discrete emotional categories," Behavior Research Methods, vol. 40, no. 1, pp. 315-321, 2008.

[30] A. Greco, G. Valenza, L. Citi, and E. P. Scilingo, "Arousal and valence recognition of affective sounds based on electrodermal activity," IEEE Sensors Journal, 2016.

[31] P. Shi, Y. Zhu, J. Allen, and S. Hu, "Analysis of pulse rate variability derived from photoplethysmography with the combination of lagged poincaré plots and spectral characteristics," Medical engineering \& physics, vol. 31, no. 7, pp. 866-871, 2009.

[32] B. Roy, R. Choudhuri, A. Pandey, S. Bandopadhyay, S. Sarangi, and S. K. Ghatak, "Effect of rotating acoustic stimulus on heart rate 
variability in healthy adults," The open neurology journal, vol. 6, p. 71, 2012.

[33] P. Guzik, J. Piskorski, T. Krauze, R. Schneider, K. H. Wesseling, A. Wykretowicz, and H. Wysocki, "Correlations between the poincare plot and conventional heart rate variability parameters assessed during paced breathing," The Journal of Physiological Sciences, vol. 57, no. 1, pp. 63-71, 2007.

[34] J. Piskorski and P. Guzik, "Filtering poincare plots," Computational methods in science and technology, vol. 11, no. 1, pp. 39-48, 2005.

[35] H.-T. Wu, P.-C. Hsu, C.-K. Sun, H.-J. Wang, C.-C. Liu, H.-R. Chen, A.B. Liu, C.-J. Tang, and M.-T. Lo, "Assessment of autonomic dysfunction in patients with type 2 diabetes using reactive hyperemia," Journal of theoretical biology, vol. 330, pp. 9-17, 2013.

[36] J. Mateo and P. Laguna, "Improved heart rate variability signal analysis from the beat occurrence times according to the ipfm model," IEEE Transactions on Biomedical Engineering, vol. 47, no. 8, pp. 985-996, 2000.

[37] M. Orini, R. Bailón, L. Mainardi, and P. Laguna, "Synthesis of hrv signals characterized by predetermined time-frequency structure by means of time-varying arma models," Biomedical Signal Processing and Control, vol. 7, no. 2, pp. 141-150, 2012.

[38] D. Schäfer, M. Nil, D. Herzig, P. Eser, H. Saner, and M. Wilhelm, "Good reproducibility of heart rate variability after orthostatic challenge in patients with a history of acute coronary syndrome," Journal of electrocardiology, vol. 48, no. 4, pp. 696-702, 2015.

[39] S. Guzzetti, M. T. La Rovere, G. D. Pinna, R. Maestri, E. Borroni, A. Porta, A. Mortara, and A. Malliani, "Different spectral components of $24 \mathrm{~h}$ heart rate variability are related to different modes of death in chronic heart failure," European heart journal, vol. 26, no. 4, pp. 357-362, 2005.

[40] J. Pan and W. J. Tompkins, "A real-time qrs detection algorithm," Biomedical Engineering, IEEE Transactions on, no. 3, pp. 230-236, 1985.

[41] M. P. Tarvainen, J.-P. Niskanen, J. A. Lipponen, P. O. Ranta-Aho, and P. A. Karjalainen, "Kubios hrv-heart rate variability analysis software," Computer methods and programs in biomedicine, vol. 113, no. 1, pp. 210-220, 2014.

[42] M. Nardelli, G. Valenza, A. Greco, A. Lanata, and E. P. Scilingo, "Recognizing emotions induced by affective sounds through heart rate variability," IEEE Transactions on Affective Computing, vol. 6, no. 4, pp. 385-394, 2015.

[43] J. A. Russell, "A circumplex model of affect." Journal of personality and social psychology, vol. 39, no. 6, p. 1161, 1980.

[44] J. Posner, J. A. Russell, and B. S. Peterson, "The circumplex model of affect: An integrative approach to affective neuroscience, cognitive development, and psychopathology," Development and psychopathology, vol. 17, no. 03, pp. 715-734, 2005.

[45] J. Mateo and P. Laguna, "Analysis of heart rate variability in the presence of ectopic beats using the heart timing signal," IEEE Transactions on Biomedical Engineering, vol. 50, no. 3, pp. 334-343, 2003.

[46] J. H. Zar, "Significance testing of the spearman rank correlation coefficient," Journal of the American Statistical Association, vol. 67, no. 339, pp. 578-580, 1972.

[47] H. W. Lilliefors, "On the kolmogorov-smirnov test for normality with mean and variance unknown," Journal of the American Statistical Association, vol. 62, no. 318, pp. 399-402, 1967.

[48] M. Memedi, D. Nyholm, A. Johansson, S. Pålhagen, T. Willows, H. Widner, J. Linder, and J. Westin, "Validity and responsiveness of athome touch screen assessments in advanced parkinson's disease," IEEE journal of biomedical and health informatics, vol. 19, no. 6, pp. 18291834, 2015.

[49] J. M. Bland and D. Altman, "Statistical methods for assessing agreement between two methods of clinical measurement," The lancet, vol. 327, no. 8476, pp. 307-310, 1986.

[50] J. M. Bland and D. G. Altman, "Measuring agreement in method comparison studies," Statistical methods in medical research, vol. 8, no. 2, pp. 135-160, 1999.

[51] M. Hollander, D. A. Wolfe, and E. Chicken, Nonparametric statistical methods. John Wiley \& Sons, 2013.

[52] R. O. Duda, P. E. Hart, and D. G. Stork, Pattern classification. John Wiley \& Sons, 2012.

[53] S. Srivastava, M. R. Gupta, and B. A. Frigyik, "Bayesian quadratic discriminant analysis," Journal of Machine Learning Research, vol. 8, no. Jun, pp. 1277-1305, 2007.

[54] M. Brennan, M. Palaniswami, and P. Kamen, "Do existing measures of poincare plot geometry reflect nonlinear features of heart rate variabil- ity?" IEEE transactions on biomedical engineering, vol. 48, no. 11, pp. 1342-1347, 2001.

[55] A. L. Roque, V. E. Valenti, H. L. Guida, M. F. Campos, A. Knap, L. C. M. Vanderlei, L. L. Ferreira, C. Ferreira, and L. C. d. Abreu, "The effects of auditory stimulation with music on heart rate variability in healthy women," Clinics, vol. 68, no. 7, pp. 960-967, 2013.

[56] S. A. F. da Silva, H. L. Guida, A. M. dos SantosAntônio, L. C. M. Vanderlei, L. L. Ferreira, L. C. de Abreu, F. H. Sousa, and V. E. Valenti, "Auditory stimulation with music influences the geometric indices of heart rate variability in men," International archives of medicine, vol. 7, no. 1 , p. $27,2014$.

[57] B. C. de Castro, H. L. Guida, A. L. Roque, L. C. de Abreu, C. Ferreira, R. S. Marcomini, C. B. Monteiro, F. Adami, V. F. Ribeiro, F. L. Fonseca et al., "Auditory stimulation with music influences the geometric indices of heart rate variability in response to the postural change maneuver," Noise and Health, vol. 16, no. 68, p. 57, 2014.

[58] L. Mourot, M. Bouhaddi, S. Perrey, S. Cappelle, M.-T. Henriet, J.-P. Wolf, J.-D. Rouillon, and J. Regnard, "Decrease in heart rate variability with overtraining: assessment by the poincare plot analysis," Clinical physiology and functional imaging, vol. 24, no. 1, pp. 10-18, 2004.

[59] P. W. Kamen, H. Krum, and A. M. Tonkin, "Poincare plot of heart rate variability allows quantitative display of parasympathetic nervous activity in humans," Clinical science, vol. 91, no. 2, pp. 201-208, 1996. 